\title{
The Latin American Society for Pediatric Research MEMBERSHIP LIST 1981-1982 Active and Honorary
}

ALVARe2, M.L., M.D., Macul 5540, Casilla 15138
Santiago il, Chile, Ph.: 2214558 ARAYA Q., M., M.D., Casilla 15138 Santiago 11,
Chile

ARIAS, G., M.D., (H.M.), Cordero 410, Quito, Ecuador

BALLESTER C., D., M.D., Casilla 15138 Santiago 11, Chile, Ph.: 2215893

RARBIERI, M.A., M.D., R. Sao Jose 1937, 14100 Ribeirao Preto, Brasil, Ph.: (0166) 252752 BAPMACH DE N., A.I., M.D., Av. Forest $3353^{\circ}$ A, 1427 Cap. Federal, Argentina, Ph. : 540775

BAPaNINI, B.M., M.D., Beruti $3507 / 7^{\circ}$ A, 1425 Buenos Aires, Argentina

BASABE, J.C., M.D., Alsina, 795 Adrogué, Prov. Buenos Aires, Argentina, Ph.: 2945537

BauzA, C.A., M.D., (H.M.), Rambla Rca. del Peru 1371, Montevideo, Uruguay

BEAS F., F., M.D., (H.M.), Casilla 5370 Correo 3, Santiago, Chile

BECU, L., M.D., (H.M.), Billinghurst 2135 p. 9/B Buenos Aires, Argentina

BERGADA, C., M.D., (H.M.), Talcakuano $11754^{\circ}$ A, guenos Aires 1013, Argentina, Ph.: 447721

BOTIINI, E.E., M.D., Araoz 2305- 4B, Buenos Aires 1425, Argentina, Ph.: 72-8213

BRAIER, J.L., M.D., Melincue 2476 Piso 5 Dto. C, Buenos Aires 1417, Argentina, Ph.: 865819

BRUNSER, O., M.D., Casilla 15138 Santiago 11 Santiago, Chile, Ph.: 2215838

BUSTOS, J.R., M.D., Hosp. das Clin. piso 16 , Casilla 627, uontevideo, Unuglay

CARALIERD, C.L., M.D., Casilla de Correo 1830, Assunción, Paraquay, Ph.: 63410

CALETTI, M.G., M.D., Gallo 1330, Buenos Aires, Argentina

CAMPOS, J.V.M., M.D., (H.M.), R. Silvia 276, 01331 S. Paulo, Brasil, Ph.: 2846611/8535705

CARDOSO, A.L., R. Oscar Freire 1360/11, 05409 S. Paulo, Brasil, Ph.: 2806898

Jair ZAZA, F.R., M.D., Inst. da Crianca, Av. Eneas de Carvalho Aguiar s/n 05403 S. Paulo, Brasil, Ph. : 2831147

CASTILIO, C.E., M.D., Eliodoro Yárez 922/75, Santiago, Chile

Cir, I., M.D., (H.M.), R. Capitao Souza Franco 208. 3000 Curitiba, Brasil, Ph.: 239040

CEIEDON G., J.M., M.D., Casilla 15138 Santiago 11, Santiago, Chile, Ph.: 2215893

CERIANI, C., J.M., M.D., San Jose $6903^{\circ}$ F, Buenos Aires 1070, Argentina

Coco, R., M.D., Cordoba $2650-1^{\circ}$ A, Buenos Aires, Argentina, Ph.: 722723

CORDANO, A., M.D., Mead Johnson Co., 47721 Evans" ville, Indiava USA

CORDERO, M.E., M.D., Dept. de Ciencias Básicas, Facultad de Med., Div. Sur - U. de Chile, Santiago, Chile Cusmansky, M., M.D., (H.M.), Calle $47-\mathrm{N}^{\circ} 388$,
La Plata 1900, Axgentina, Ph.: 37782

LAVILA, N., M.D., Ave. America 3351, Quito, Ecuador DE LOS SANTOS, F., M.D., Juan Paullier 2014, Montevideo, Uruguay, Ph.: 415976

DI MARTINO, C., M.F., Edificio Flening, Coronel Bogado 1043, Assumcao, Paraguay, Ph.: 63205

DIAMENL, A.J., M.D., Av. Angélica 1832, 01228 S. Paulo, Brasil, Ph. 2561133

DRur, R., M.D., Calle 11 № 718, 1900 La Plata, Argentina, Ph.: 31084

DUJONNE, I.L., M.D., Hosp. Ped. Pedro de Elizalde, Montes de Oca, 40, Buenos Aires, Argentina

ESPINOZA, J.M., M.D., Monseñor Escribá de Balaguer
8976, La condes, Santiago, Chile, Ph.: 2214030

EXENI, R.A., M.D., Calle Diaz Velez 504, La Lucila (1636), Buenos Aires, Argentina

FAGUNDES NETO, U., M.D., R. BOtucatu 720, $04023 \mathrm{~S}$ Paulo, Brasil, Ph.: 5444255

FAHRER, M., M.D., Salguero $24843^{\circ}$ A, Buenos Aires, Argentina

FEIOMAN, G., M.D., Samiento $4312-2^{\circ}$ B, Buenos Aires, Axgentina

FERRARIS, J.R., M.D., Gascon 450, Buenos Aires, Argentina

FERREYRA, E.R.S., M.D., Calilegua 147 - v. Martha, Cordoba, Argentin

FREILIG, H., M.D., Lab. Virología Hosp. de Niños, Gallo 1330, Buenos Aires, Argentina, Ph. : 866831 Ext. 37

FALLO, G., M.D., Viamonte $16474^{\circ} 17,1055$ Buenos Aires, Argentina, Ph.: 414646

JARDNER, L.I., M.D., (H.M.), Dept. of Ped., Upstate Med. Sch., 750 E. Adams St., Syracuse, NY

GLANANIONIO, C., M.D., (H.M.), Dept. of Ped., Hosp. Italiano, Gascon 450 - Martinez, Buenos Aires,

GIUSSI, G.A., M.D., Centro Latinoanericano de Per. Desarrollo Humano, Casilla Correo 627, Montey Desarrollo Humano, Casilla
video, Uruguay, Ph.: $\mathbf{4 1 0 2 8 9}$

GOOARD, C., M.D., (H.M.), Regional HOSp., 1870 Monthey, Suiza, Ph.: 2570611

Grupgerg, J., M.D., Casilla de Correo 1138, Mantevideo, Urugray, Ph.: 791266

GRUNFEID, B., M.D., Lafinur 3291, 1425 Buenos Aires, Argentina

GUZMAN, M.T., M.D., Isidora Coyanechea 3048/303 Las Condes, Santiago, Chile, Ph.: 483261

HEINRICH, J.J., M.D. , Conde 1757, 1426 Buenos Aires, Argentina, Ph.: 866831/874605

HERRESI, G., M.D., NNTA. Casilla 15138 Santiago 11 , Chile, Ph.: 221 ' 5962

IOFCANSKY, S., M.D., Lambaré 1095-16, Buenos Aires, Argentina, Ph.: $866831 / 874605$

JASPER, G.H., M.D., Viamonte 2909 - 14 A, Buenos Aires, Argentina, Ph.: 880113

KNOPF, C.F., M.D., Viamonte 1331 ler. piso, 1053 Buenos Aires, Argentina, Ph. 408350

LACASSIE, Y., Y.D., DNAA. Casilla 15138 Santiago 11, Chile, Ph.: 2211304

IARGUIA, M., M.D., Larrea $14741^{\circ}$ A, Buenos Aires, Argentina

LAZZARI, M.E.E., M.D., Pueyrredon 1655 P. 13 Dto. B, Buenos Aires, Argentina

IEJARRACA, H.A., M.D., Juncal $28512^{\circ} 6$, Buenos Aires, Argentina

LEVIN, G.M., M.D., Billinghurst $15758^{\circ}$ Piso Dpto. B, Buenos Aires, Argentina, Ph.: 8263701

LLANOS, A.J., M.D., Casilla 16038, Santiago 9, Chile, Ph.: 741560

LOZANO Z., U., M.D., (H.M.), Av. Petit Thouars 3125

URETKIN, A., M.D., (H.M.), Belgrano 287, Rio Oarto, Argentina, Ph.: 353451

MANTEROLA, A.C., M.D., Mendoza 5131, Buenos Aires 1431, Argentina

MARCONDES, E., M.D., (H.M.), Clin. Ped., Hosp. das

MA22A, C.S., M.D., Canning $24522^{\circ} 5$, Buenos Aires, Argentina, Ph.: 725411

MENDILAHARZU, F., M.D., Las Heras 3702 48-1425, Buenos Aires, Argentina

MaNDILAFARZU, H., M.D., Cabello 3978, Piso 3, Dept. 6, Buenos Aires, Argentina, Ph.: 727876

MENDILAHARZU, J.L., M.D., Libertad 1559, Buenos Ajres, Argentina, Ph.: 44679
MERA, J.A., M.D., Malabia 2777, 1425 Buenos Aires,
Argentina

MaNCKERERG, F., M.D., (H.M.), Casilla 5370, Santiago, Chile, Ph. : 221-4030

MOTA, F., M.D., Hosp. Infantil de México, Marquez 162, México DF, Mexico, Ph.: 7610333

MaNTEIRO, D., M.D., (H.M.), Serv. de Anatomia Pathol., Faculdade de Med.-SP, 05403 S. Paulo,

MORASSO, M.C., M.D., Zeballos 5851, 1875 wilde, Buenos Aires, Argentina, Ph.: 207765

Muzzo, S.B., M.D., Las Nipas 3952, Las Condes, Santiago, Chile, Ph.: 2214922

iNEISan, W., M.D., (H.M.), 3300 Henry Ave., Philadelphia, PA 19129 USA

NICHOLS, B.L., M.D., (H.M.), 9917 Oboe St., Houston,

NOVOA, F.S., M.D., Hosp. Manuel Arriarán, Hernando de Aguirre 1183, Santiago, Chile

O'DONNELL, A., M.D., Córdoba 803, Buenos Aires, Argentina

OLIVARES PIAZA, J.R., M.D., Casilla 2340, La Paz, Bolivia, Ph.: 29347

OLIVARES, M.A., M.D., Casilla 15138 santiago 11, Chile, Ph.: 2215962

OITVEROS D., M.A., M.D., Rio Moche 371, Pueblo Libre (21), Lima, Peri, Ph.: 611872

ORDWAY, N., (H.M.), 2201 E. Nicolet Ave., Phoenix, AZ 85020 USA

ORTI2, W.R., M.D., Av. Miraflores 11-17, Ambato, Ecrador

CSORIO, A., M.D., Casilla 10677 Distrito 1, Montevideo, Uruguay

OMMAN, S., M.D., (H.M.), Casilla 15138, Santiago 11, Santiago, Chile, Ph.: 2214105

PAPENDIEK, L.G, M.D., Gallo 1330, 1425 Buenos Aires, Argentina, Ph.: 7820597

PENCHANSKY, L., M.D., Children's HoSp. of Pitts. 125 Desoto st., Pittsburgh, PA 15123 USA

PENA, J.L., M.D., Siria 6295, Montevideo, Uruguay, $\mathrm{Ph}: 501189$

PENALVER, A., M.D., (H.M.), Austria 2258, Buenos Aires, Argentina

PENCHASZADEH, V.B., M.D., Prof. de Genet. Med., Univ. Ctr. de Venezuela, Apartado 50647, Caracas 1050 A, venezuela, Ph. : 616712/770581

RIOS, E., M.D., E. Recabarren 2348, Santiago, Chile

ROCHA, R., M.D., (H.M.), R. Delfin Moreira 952/101, 22241 Río de Janeiro, Brasil, Ph.: (021) 2749127/

ROSEMBERG, S., M.D., R. Ferreira de Araujo 961/172, 05428 S. Pawlo, Brasil, Ph.: 2128467

SACANAN, E., M.D., Alibarellos 1551, 1640 Martinez P.B.A., Argentina

SCHIESINGER, L., M.D., Casilla 15138 Santiago 11, Chile, Ph. 221596

SCrMIDT, B.J., M.D., Cardoson de Almeida 2144, 01251 S. Paulo, Brasil, Ph.: 624062

SETIAN, N.. M.D., R, Volumtarios da Patria 2297 $2^{\circ}, 02011$ S. Paulo, Brasil, Ph.: 2980742

Saro, E.T., M.D., Aguero 2443, 2425 Bueinos Aires, Argentina

SOLA, H.A., M.D., Av. Dr. Honorio Pueyrredon 1455 $4^{\circ}$ Piso Dept. 8, 1414 Buenos Aires, Argentina,
Ph.: 545145

(H.M.) Honorary Member

SORDELLI, D.O., M.D., 130 Gorman Bldg., Intematl. Ctr., Interdisciplinary Studies of Immurnology, Georgetown Univ. Hosp.: 3800 Peservoir Rd.,
Washington, D.C. 20007, Ph.: (202) 625-7740 SPADA, R.P., M.D., General Paz 147, Cordoba, Argentina 
SPENCER, E., M.D., Virol. Lab. Div. of Basic Sciences, IVTA. Casilla 15138 Santiago 11, Chile

SPEROTrO, G., M.D., Inst. da Crianca, Av, theas de carvalho Aguar s/n ${ }^{\circ}, 05403$ s. Paulo, Brasil Ph.: (011) 8816311

STEKEL, A., M.D., Casilla 15138 Santiago 11, Chile, Ph.: 221 S962

TOBOADA, C.R.S., M.D., Inst. Genet. thomana - UMSA, Casilla 2430, La Paz, Bolivia, Ph.: 356822
mABOADA L., J.M.G., M.D., Casilla 3050, La Paz, Bolivia, Ph.: 353071

TERAN PUENEE, J., M.D., 12 de Octubre 622, P.O. Box 3711, Quito, Ecuador

TORIN, B., M.D., INCAP, Apartado Postal 1188, Gutemala, Guatemala
UAUY, R., M.D., Casilla 15138 Santiago 11, Chile, Ph.: 2216337

VOYER, L., M.D., Ramallo 2050, Buenos Aires,

Argentina

zEIAzKo, M.E., M.D., Calle Echeverría 2109, Buenos Aires, Argentina 